\title{
On the Modularity of Deciding Call-by-Need
}

\author{
Irène Durand ${ }^{1}$ and Aart Middeldorp ${ }^{2 \star}$ \\ 1 Université de Bordeaux I \\ 33405 Talence, France \\ idurand@labri.u-bordeaux.fr \\ 2 Institute of Information Sciences and Electronics \\ University of Tsukuba, Tsukuba 305-8573, Japan \\ ami@is.tsukuba.ac.jp
}

\begin{abstract}
In a recent paper we introduced a new framework for the study of call by need computations. Using elementary tree automata techniques and ground tree transducers we obtained simple decidability proofs for a hierarchy of classes of rewrite systems that are much larger than earlier classes defined using the complicated sequentiality concept. In this paper we study the modularity of membership in the new hierarchy. Surprisingly, it turns out that none of the classes in the hierarchy is preserved under signature extension. By imposing various conditions we recover the preservation under signature extension. By imposing some more conditions we are able to strengthen the signature extension results to modularity for disjoint and constructor-sharing combinations.
\end{abstract}

\section{Introduction}

The seminal work of Huet and Lévy 9 on optimal normalizing reduction strategies for orthogonal rewrite systems marks the beginning of the quest for decidable subclasses of (orthogonal) rewrite systems that admit a computable call by need strategy for deriving normal forms. Call by need means that the strategy may only contract needed redexes, i.e., redexes that are contracted in every normalizing rewrite sequence. Huet and Lévy showed that for the class of orthogonal rewrite systems every term not in normal form contains a needed redex and repeated contraction of needed redexes results in a normal form if the term under consideration has a normal form. However, neededness is in general undecidable. In order to obtain a decidable approximation to neededness Huet and Lévy introduced in the second part of [9] the subclass of strongly sequential systems. In a strongly sequential system at least one of the needed redexes in every reducible term can be effectively computed. Moreover, Huet and Lévy showed that strong sequentiality is a decidable property of orthogonal rewrite systems. Several authors ([26]10]13|15|17|20|19]) studied extensions of the class of strong sequential rewrite systems that preserve its good properties.

\footnotetext{
* Partially supported by the Grant-in-Aid for Scientific Research C(2) 11680338 of the Ministry of Education, Science, Sports and Culture of Japan.
} 
In a previous paper (Durand and Middeldorp [6]) we presented a uniform framework for decidable call by need based on approximations. We introduced classes $\mathcal{C B N} \mathcal{N}_{\alpha}$ of rewrite systems parameterized by approximation mappings $\alpha$. In [6] we identified the properties an approximation mapping $\alpha$ has to satisfy in order that the resulting class $\mathcal{C B N}_{\alpha}$ is decidable and every rewrite system in that class admits a computable normalizing call by need strategy. We showed moreover that our classes are much larger than the corresponding classes based on the difficult sequentiality concept.

Not much is known about the complexity of the problem of deciding membership in one of the classes that guarantees a computable call by need strategy to normal form. Comon [2] showed that strong sequentiality of a left-linear rewrite system can be decided in exponential time. Moreover, for left-linear rewrite systems satisfying the additional syntactic condition that whenever two proper subterms of left-hand sides are unifiable one of them matches the other, strong sequentiality can be decided in polynomial time. The class of forward-branching systems (Strandh [18]), a proper subclass of the class of orthogonal strongly sequential systems, coincides with the class of transitive systems (Toyama et al. [21]) and can be decided in quadratic time (Durand [5]). For classes higher in the hierarchy only double exponential upper bounds are known ([7]).

Consequently, it is of obvious importance to have results available that enable to split a rewrite system into smaller components such that membership in $\mathcal{C} \mathcal{B N}_{\alpha}$ of the components implies membership of the original system in $\mathcal{C} \mathcal{B N}_{\alpha}$. Such modularity results have been extensively studied for basic properties like confluence and termination, see 81416] for overviews.

The simplest kind of modularity results are concerned with enriching the signature. Most properties of rewrite systems are preserved under signature extension. Two notable exceptions are the normal form property and the unique normal form property (with respect to reduction), see Kennaway et al. 11]. Also some properties dealing with ground terms are not preserved under signature extension. For instance, consider the property that every ground term has a normal form, the rewrite system consisting of the single rewrite rule $\mathrm{f}(x) \rightarrow \mathrm{f}(x)$, and add a new constant a. (A slightly more interesting example is obtained by adding the rewrite rule $\mathrm{f}(\mathrm{b}) \rightarrow \mathrm{b}$.) It turns out that for no $\alpha$ membership in $\mathcal{C} \mathcal{B N}_{\alpha}$ is preserved under signature extension. In this paper we present several sufficient conditions which guarantee the preservation of signature extension. These results are presented in Section 3 .

Since preservation under signature extension does not give rise to a very useful technique for splitting a system into smaller components, in Section 4 we consider combinations of systems without common function symbols as well as constructor-sharing combinations.

In the next section we briefly recall the framework of our earlier paper [6] for analyzing call by need computations in term rewriting and we introduce some useful definitions. 


\section{Preliminaries}

We assume the reader is familiar with the basics of term rewriting $([1,412])$. We recall the following definitions from [6]. We refer to the latter paper for motivation and examples. A term rewrite system (TRS for short) consists of rewrite rules $l \rightarrow r$ that satisfy $\operatorname{root}(l) \notin \mathcal{V}$ and $\mathcal{V} \operatorname{ar}(r) \subseteq \mathcal{V}$ ar $(l)$. If the second condition is not imposed we find it useful to speak of extended TRSs (eTRSs). Such systems arise naturally when we approximate TRSs, as explained below.

Let $\mathcal{R}$ be an eTRS over a signature $\mathcal{F}$. The set of ground normal forms of $\mathcal{R}$ is denoted by $\operatorname{NF}(\mathcal{R}, \mathcal{F})$. Let $\mathcal{R} \bullet$ be the eTRS $\mathcal{R} \cup\{\bullet \rightarrow \bullet\}$ over the extended signature $\mathcal{F}_{\bullet}=\mathcal{F} \cup\{\bullet\}$. We say that redex $\Delta$ in $C[\Delta] \in \mathcal{T}(\mathcal{F})$ is $\mathcal{R}$-needed if there is no term $t \in \operatorname{NF}(\mathcal{R}, \mathcal{F})$ such that $C[\bullet] \rightarrow_{\mathcal{R}}^{*} t$. So to determine $\mathcal{R}$ neededness of a redex $\Delta$ in $C[\Delta]$ we replace it by $\bullet$ and check whether we can derive a normal form without $\bullet$. Redex $\Delta$ is $\mathcal{R}$-needed only if this is impossible. Note that $\operatorname{NF}(\mathcal{R}, \mathcal{F})=\operatorname{NF}\left(\mathcal{R}_{\bullet}, \mathcal{F}_{\bullet}\right)$. For orthogonal TRSs $\mathcal{R}$-neededness coincides with neededness. We denote by $\operatorname{WN}(\mathcal{R}, \mathcal{F})$ the set of all ground terms in $\mathcal{T}(\mathcal{F})$ that rewrite in $\mathcal{R}$ to a normal form in $\operatorname{NF}(\mathcal{R}, \mathcal{F})$. If no confusion can arise, we just write $\operatorname{WN}(\mathcal{R})$.

Let $\mathcal{R}$ and $\mathcal{S}$ be eTRSs over the same signature $\mathcal{F}$. We say that $\mathcal{S}$ approximates $\mathcal{R}$ if $\rightarrow_{\mathcal{R}}^{*} \subseteq \rightarrow_{\mathcal{S}}^{*}$ and $\operatorname{NF}(\mathcal{R}, \mathcal{F})=\operatorname{NF}(\mathcal{S}, \mathcal{F})$. An approximation mapping is a mapping $\alpha$ from TRSs to eTRSs with the property that $\alpha(\mathcal{R})$ approximates $\mathcal{R}$, for every TRS $\mathcal{R}$. In the following we write $\mathcal{R}_{\alpha}$ instead of $\alpha(\mathcal{R})$. The class of left-linear TRSs $\mathcal{R}$ such that every reducible term in $\mathcal{T}(\mathcal{F})$ has an $\mathcal{R}_{\alpha}$-needed redex is denoted by $\mathcal{C B N}_{\alpha}$. Here $\mathcal{F}$ denotes the signature of $\mathcal{R}$. We assume throughout the paper that ground terms exist. Although not explicitly stated in our earlier paper [6] we assume that $\mathcal{R}$ is a proper TRS (i.e., not an eTRS). For arbitrary left-linear eTRSs $\mathcal{R}$ we introduce a corresponding class $\mathcal{C B N}$ which contains the eTRSs with the property that every reducible ground term has an $\mathcal{R}$-needed redex. So a TRS $\mathcal{R}$ belongs to $\mathcal{C} \mathcal{B N}_{\alpha}$ if and only if $\mathcal{R}_{\alpha} \in \mathcal{C B N}$.

Next we define the approximation mappings s, nv, and g. Let $\mathcal{R}$ be a TRS. The strong approximation $\mathcal{R}_{\mathrm{s}}$ is obtained from $\mathcal{R}$ by replacing the right-hand side of every rewrite rule by a variable that does not occur in the corresponding left-hand side. The $n v$ approximation $\mathcal{R}_{\mathrm{nv}}$ is obtained from $\mathcal{R}$ by replacing the variables in the right-hand sides of the rewrite rules by pairwise distinct variables that do not occur in the corresponding left-hand sides. An eTRS is called growing if for every rewrite rule $l \rightarrow r$ the variables in $\mathcal{V} \operatorname{ar}(l) \cap \mathcal{V} \operatorname{ar}(r)$ occur at depth 1 in $l$. The growing approximation $\mathcal{R}_{\mathrm{g}}$ is defined as any growing eTRS that is obtained from $\mathcal{R}$ by renaming the variables in the right-hand sides that occur at a depth greater than 1 in the corresponding left-hand sides. In [6] we showed that for a left-linear TRS $\mathcal{R}$ and $\alpha \in\{\mathrm{s}, \mathrm{nv}, \mathrm{g}\}$ membership of $\mathcal{R}$ in $\mathcal{C B N}_{\alpha}$ is decidable.

We conclude this preliminary section with some easy definitions. A rewrite rule $l \rightarrow r$ of an eTRS is collapsing if $r$ is a variable. A redex with respect to a collapsing rewrite rule is also called collapsing and so is an eTRS that contains a collapsing rewrite rule. A redex is called flat if it does not contain smaller redexes. Let $\mathcal{R}$ be a TRS over the signature $\mathcal{F}$. A function symbol in $\mathcal{F}$ is called 
defined if it is the root symbol of a left-hand side of a rewrite rule in $\mathcal{R}$. All other function symbols in $\mathcal{F}$ are called constructors. A term without defined symbols is called a constructor term. We say that $\mathcal{R}$ is a constructor system (CS for short) if the arguments of the left-hand side of a rewrite rule are constructor terms.

Let $\mathcal{R}$ be an eTRS over the signature $\mathcal{F}$ and let $\mathcal{G} \subseteq \mathcal{F}$. We denote by $\operatorname{WN}(\mathcal{R}, \mathcal{F}, \mathcal{G})$ the set of terms in $\mathcal{T}(\mathcal{G})$ that have a normal form with respect to $(\mathcal{R}, \mathcal{F})$. The subset of $\operatorname{WN}(\mathcal{R}, \mathcal{F}, \mathcal{G})$ consisting of those terms that admit a normalizing rewrite sequence in $(\mathcal{R}, \mathcal{F})$ containing a root rewrite step is denoted by $\operatorname{WNR}(\mathcal{R}, \mathcal{F}, \mathcal{G})$. If $\mathcal{F}=\mathcal{G}$ then we just write $\operatorname{WNR}(\mathcal{R}, \mathcal{F})$ or even $\operatorname{WNR}(\mathcal{R})$ if the signature is clear from the context. We also find it convenient to write $\operatorname{WN}_{\bullet}(\mathcal{R}, \mathcal{F}, \mathcal{G})$ for $\operatorname{WN}\left(\mathcal{R}_{\bullet}, \mathcal{F}_{\bullet}, \mathcal{G}_{\bullet}\right)$ and $\operatorname{WNR}_{\bullet}(\mathcal{R}, \mathcal{F}, \mathcal{G})$ for $\operatorname{WNR}\left(\mathcal{R}_{\bullet}, \mathcal{F}_{\bullet}, \mathcal{G}_{\bullet}\right)$. A reducible term without needed redexes is called free. A minimal free term has the property that its proper subterms are not free.

\section{Signature Extension}

In this section we study the question whether membership in $\mathcal{C B N}_{\alpha}$ is preserved after adding new function symbols.

Definition 1. We say that a class $\mathcal{C}$ of eTRSs is preserved under signature extension if $(\mathcal{R}, \mathcal{G}) \in \mathcal{C}$ for all $(\mathcal{R}, \mathcal{F}) \in \mathcal{C}$ and $\mathcal{F} \subseteq \mathcal{G}$.

The results we obtain in this section are summarized below. In the result marked with $(*)$ signature extension only holds if we further require that the set of $\mathcal{R}_{\mathrm{nv}}$-normalizable terms over the original signature is not increased.

\begin{tabular}{llc} 
approximation & \multicolumn{1}{c}{ sufficient conditions } & Theorem \\
\hline strong & $\exists$ ground normal form & 1 \\
nv & $\exists$ external normal form \\
nv & $\mathcal{R}$ is collapsing or $\mathcal{R}$ is a CS $(*)$ \\
growing & $\exists$ external normal form
\end{tabular}

All our proofs follow the same strategy. We consider a TRS $\mathcal{R}$ over a signature $\mathcal{F}$ such that $(\mathcal{R}, \mathcal{F}) \in \mathcal{C B} \mathcal{N}_{\alpha}$. Let $\mathcal{G}$ be an extension of $\mathcal{F}$. Assuming that $(\mathcal{R}, \mathcal{G}) \notin$ $\mathcal{C B N}_{\alpha}$, we consider a minimal $\left(\mathcal{R}_{\alpha}, \mathcal{G}\right)$-free term $t$ in $\mathcal{T}(\mathcal{G})$. By replacing the maximal subterms of $t$ that start with a function symbol in $\mathcal{G} \backslash \mathcal{F}$ - such subterms will be called aliens or more precisely $\mathcal{G} \backslash \mathcal{F}$-aliens in the sequel-by a suitable term in $\mathcal{T}(\mathcal{F})$, we obtain an $\left(\mathcal{R}_{\alpha}, \mathcal{F}\right)$-free term $t^{\prime}$ in $\mathcal{T}(\mathcal{F})$. Hence $(\mathcal{R}, \mathcal{F}) \notin \mathcal{C B} \mathcal{N}_{\alpha}$, contradicting the assumption.

Our first example shows that $\mathcal{C B N}$ is not preserved under signature extension.

Example 1. Consider the TRS

$$
\mathcal{R}=\left\{\begin{aligned}
\mathrm{f}(x, \mathrm{~g}(y), \mathrm{h}(z)) & \rightarrow x \\
\mathrm{f}(\mathrm{h}(z), x, \mathrm{~g}(y)) & \rightarrow x \\
\mathrm{f}(\mathrm{g}(y), \mathrm{h}(z), x) & \rightarrow x \\
\mathrm{a} & \rightarrow \mathrm{a}
\end{aligned}\right\}
$$


over the signature $\mathcal{F}$ consisting of all symbols appearing in the rewrite rules. As $\operatorname{NF}(\mathcal{R}, \mathcal{F})=\varnothing$, trivially $(\mathcal{R}, \mathcal{F}) \in \mathcal{C B} \mathcal{N}_{\text {s }}$. Let $\mathcal{G}=\mathcal{F} \cup\{\mathrm{b}\}$ with b a constant. We have $(\mathcal{R}, \mathcal{G}) \notin \mathcal{C} \mathcal{B N}_{\mathrm{s}}$ as the term $\mathrm{f}(\mathrm{a}, \mathrm{a}, \mathrm{a})$ has no $\left(\mathcal{R}_{\mathrm{s}}, \mathcal{G}\right)$-needed redex:

$$
\begin{aligned}
& \mathrm{f}(\bullet, \mathrm{a}, \mathrm{a}) \rightarrow_{\mathrm{s}} \mathrm{f}(\bullet, \mathrm{g}(\mathrm{a}), \mathrm{a}) \rightarrow_{\mathrm{s}} \mathrm{f}(\bullet, \mathrm{g}(\mathrm{a}), \mathrm{h}(\mathrm{a})) \rightarrow_{\mathrm{s}} \mathrm{b} \\
& \mathrm{f}(\mathrm{a}, \bullet, \mathrm{a}) \rightarrow_{\mathrm{s}} \mathrm{f}(\mathrm{h}(\mathrm{a}), \bullet, \mathrm{a}) \rightarrow_{\mathrm{s}} \mathrm{f}(\mathrm{h}(\mathrm{a}), \bullet, \mathrm{g}(\mathrm{a})) \rightarrow_{\mathrm{s}} \mathrm{b} \\
& \mathrm{f}(\mathrm{a}, \mathrm{a}, \bullet) \rightarrow_{\mathrm{s}} \mathrm{f}(\mathrm{g}(\mathrm{a}), \mathrm{a}, \bullet) \rightarrow_{\mathrm{s}} \mathrm{f}(\mathrm{g}(\mathrm{a}), \mathrm{h}(\mathrm{a}), \bullet) \rightarrow_{\mathrm{s}} \mathrm{b}
\end{aligned}
$$

The above example is interesting since it furthermore shows that $\mathcal{C B N}_{\mathrm{s}}$ properly includes the class of strongly sequential TRSs defined by Huet and Lévy [9], contrary to the claim in [6] that these two classes coincide.

One may wonder whether there are any nontrivial counterexamples, where nontrivial means that the set of ground normal forms is nonempty. Surprisingly, the answer is yes, provided we consider an approximation map $\alpha$ that is at least as good as nv.

Example 2. Consider the TRS

$$
\mathcal{R}=\left\{\begin{array}{lll}
\mathrm{f}(x, \mathrm{a}, \mathrm{b}) \rightarrow \mathrm{g}(x) & \mathrm{f}(\mathrm{a}, \mathrm{a}, \mathrm{a}) \rightarrow \mathrm{g}(\mathrm{a}) & \mathrm{g}(\mathrm{a}) \rightarrow \mathrm{g}(\mathrm{a}) \\
\mathrm{f}(\mathrm{b}, x, \mathrm{a}) \rightarrow \mathrm{g}(x) & \mathrm{f}(\mathrm{b}, \mathrm{b}, \mathrm{b}) \rightarrow \mathrm{g}(\mathrm{a}) & \mathrm{g}(\mathrm{b}) \rightarrow \mathrm{g}(\mathrm{b}) \\
\mathrm{f}(\mathrm{a}, \mathrm{b}, x) \rightarrow \mathrm{g}(x) & \mathrm{e}(x) & \rightarrow x
\end{array}\right\}
$$

over the signature $\mathcal{F}$ consisting of all symbols appearing in the rewrite rules. First we show that $(\mathcal{R}, \mathcal{F}) \in \mathcal{C} \mathcal{B N}_{\text {nv }}$. It is not difficult to show that the only $\left(\mathcal{R}_{\text {nv }}, \mathcal{F}\right)$ normalizable terms are $\mathrm{a}, \mathrm{b}$, and $\mathrm{e}(t)$ for every $t \in \mathcal{T}(\mathcal{F})$. Since $\mathrm{a}$ and $\mathrm{b}$ are normal forms, we only have to show that every e $(t)$ contains an $\left(\mathcal{R}_{\text {nv }}, \mathcal{F}\right)$-needed redex, which is easy since $\mathrm{e}(t)$ itself is an $\left(\mathcal{R}_{\mathrm{nv}}, \mathcal{F}\right)$-needed redex. Let $\mathcal{G}=\mathcal{F} \cup\{\mathrm{c}\}$ with c a constant. We have $(\mathcal{R}, \mathcal{G}) \notin \mathcal{C} \mathcal{B N}_{\text {nv }}$ as the term $\mathrm{f}(\mathrm{e}(\mathrm{a}), \mathrm{e}(\mathrm{a}), \mathrm{e}(\mathrm{a}))$ has no $\left(\mathcal{R}_{\mathrm{nv}}, \mathcal{G}\right)$-needed redex:

$$
\begin{aligned}
& \mathrm{f}(\bullet, \mathrm{e}(\mathrm{a}), \mathrm{e}(\mathrm{a})) \rightarrow_{\mathrm{nv}} \mathrm{f}(\bullet, \mathrm{a}, \mathrm{e}(\mathrm{a})) \rightarrow_{\mathrm{nv}} \mathrm{f}(\bullet, \mathrm{a}, \mathrm{b}) \rightarrow_{\mathrm{nv}} \mathrm{g}(\mathrm{c}) \\
& \mathrm{f}(\mathrm{e}(\mathrm{a}), \bullet, \mathrm{e}(\mathrm{a})) \rightarrow_{\mathrm{nv}} \mathrm{f}(\mathrm{b}, \bullet, \mathrm{e}(\mathrm{a})) \rightarrow_{\mathrm{nv}} \mathrm{f}(\mathrm{b}, \bullet, \mathrm{a}) \rightarrow_{\mathrm{nv}} \mathrm{g}(\mathrm{c}) \\
& \mathrm{f}(\mathrm{e}(\mathrm{a}), \mathrm{e}(\mathrm{a}), \bullet) \rightarrow_{\text {nv }} \mathrm{f}(\mathrm{a}, \mathrm{e}(\mathrm{a}), \bullet) \rightarrow_{\text {nv }} \mathrm{f}(\mathrm{a}, \mathrm{b}, \bullet) \rightarrow_{\text {nv }} \mathrm{g}(\mathrm{c})
\end{aligned}
$$

For $\alpha=\mathrm{s}$ there is no nontrivial counterexample.

Theorem 1. The subclass of $\mathcal{C B N}_{\mathrm{s}}$ consisting of all orthogonal TRSs $(\mathcal{R}, \mathcal{F})$ such that $\operatorname{NF}(\mathcal{R}, \mathcal{F}) \neq \varnothing$ is preserved under signature extension.

We refrain from giving the proof at this point since the statement easily follows from Theorem 3 below, whose proof is presented in detail. Our second result states that the subclass of $\mathcal{C B N}$ consisting of all eTRSs $\mathcal{R}$ with the property defined below is preserved under signature extension.

Definition 2. We say that an eTRS $\mathcal{R}$ has external normal forms if there exists a ground normal form which is not an instance of a proper non-variable subterm of a left-hand sides of a rewrite rule in $\mathcal{R}$. 
Note that the TRS of Example 2lacks external normal forms as both ground normal forms $a$ and $b$ appear in the left-hand sides of the rewrite rules. Further note that it is decidable whether a left-linear TRS has external normal forms by straightforward tree automata techniques. Finally note that the external normal form property is satisfied whenever there exists a constant not occurring in the left-hand sides of the rewrite rules.

Before proving our second result we present a useful lemma which is used repeatedly in the sequel.

Lemma 1. Let $\mathcal{R}$ be a left-linear eTRS. Every minimal free term belongs to $\operatorname{WNR}(\mathcal{R})$.

Proof. Let $\mathcal{F}$ be the signature of $\mathcal{R}$ and let $t \in \mathcal{T}(\mathcal{F})$ be a minimal free term. For every redex position $p$ in $t$ we have $t[\bullet]_{p} \in \mathrm{WN}_{\bullet}(\mathcal{R})$. Let $p^{\prime}$ be the minimum position above $p$ at which a contraction takes place in any rewrite sequence from $t[\bullet]_{p}$ to a normal form in $\mathcal{T}(\mathcal{F})$ and define $P=\left\{p^{\prime} \mid p\right.$ is a redex position in $\left.t\right\}$. Let $p^{*}$ be a minimal position in $P$. We show that $p^{*}=\epsilon$. If $p^{*}>\epsilon$ then we consider the term $\left.t\right|_{p^{*}}$. Let $q$ be a redex position in $\left.t\right|_{p^{*}}$. There exists a redex position $p$ in $t$ such that $p=p^{*} q$. We have $\left.t\right|_{p^{*}}[\bullet]_{q}=\left.\left(t[\bullet]_{p}\right)\right|_{p^{*}} \in \mathrm{WN}_{\bullet}(\mathcal{R})$ by the definition of $p^{*}$. Since $\left.t\right|_{p^{*}}$ has at least one redex, it follows that $\left.t\right|_{p^{*}}$ is free. As $\left.t\right|_{p^{*}}$ is a proper subterm of $t$ we obtain a contradiction to the minimality of $t$. Hence $p^{*}=\epsilon$. So there exists a redex position $p$ in $t$ and a rewrite sequence $A: t[\bullet]_{p} \rightarrow{ }_{\mathcal{R}, \mathcal{F} \bullet}^{+} u \in \operatorname{NF}(\mathcal{R}, \mathcal{F})$ that contains a root rewrite step. Because $\mathcal{R}$ is left-linear and $\bullet$ does not occur in the rewrite rules of $\mathcal{R}, \bullet$ cannot contribute to this sequence. It follows that if we replace in $A$ every occurrence of $\bullet$ by $\left.t\right|_{p}$ we obtain an $(\mathcal{R}, \mathcal{F})$-rewrite sequence from $t$ to $u$ with a root rewrite step.

In particular, minimal free terms are not root-stable.

Theorem 2. The subclass of $\mathcal{C B N}$ consisting of all left-linear eTRSs with external normal forms is preserved under signature extension.

Proof. Let $(\mathcal{R}, \mathcal{F}) \in \mathcal{C B N}$ and let $c \in N F(\mathcal{R}, \mathcal{F})$ be an external normal form. Let $\mathcal{F} \subseteq \mathcal{G}$. We have to show that $(\mathcal{R}, \mathcal{G}) \in \mathcal{C B N}$. Suppose to the contrary that $(\mathcal{R}, \mathcal{G}) \notin \mathcal{C B N}$. According to Lemma 1 there exists a term $t \in \operatorname{WNR}(\mathcal{R}, \mathcal{G})$ without $(\mathcal{R}, \mathcal{G})$-needed redex. Let $t^{\prime}$ be the term in $\mathcal{T}(\mathcal{F})$ obtained from $t$ by replacing every $\mathcal{G} \backslash \mathcal{F}$-alien by $c$. Because $t$ is not root-stable and $\mathcal{R}$ left-linear, $t^{\prime}$ must be reducible. Hence $t^{\prime}$ contains an $(\mathcal{R}, \mathcal{F})$-needed redex $\Delta$, say at position $p$. Because $c$ is an external normal form, $\Delta$ is also a redex in $t$ and hence there exists a rewrite sequence $t[\bullet]_{p} \rightarrow{ }_{\mathcal{R}, \mathcal{G}}^{+} u$ with $u \in \operatorname{NF}(\mathcal{R}, \mathcal{G})$. If we replace in this rewrite sequence every $\mathcal{G} \backslash \mathcal{F}$-alien by $c$, we obtain a rewrite sequence $t^{\prime}[\bullet]_{p} \rightarrow{ }_{\mathcal{R}, \mathcal{F}}^{+} u^{\prime}$. Because $c$ does not unify with a proper non-variable subterm of a left-hand side of a rewrite rule, it follows that $u^{\prime} \in \operatorname{NF}(\mathcal{R}, \mathcal{F})$. Hence $\Delta$ is not an $(\mathcal{R}, \mathcal{F})$-needed redex in $t^{\prime}$, yielding the desired contradiction.

Note that for $\mathcal{C B N}_{\mathrm{s}}$ the above theorem is a special case of Theorem 1 since the existence of an external normal form implies the existence of a ground normal form. 
In the remainder of this section we present a signature extension result for TRSs without external normal form. Such TRSs are quite common (see also Lemma 4 below).

Example 3. Consider the TRS

$$
\mathcal{R}=\left\{\begin{array}{ll}
0+y \rightarrow y & \mathbf{s}(x)+y \rightarrow \mathbf{s}(x+y) \\
0 \times y \rightarrow 0 & \mathbf{s}(x) \times y \rightarrow x \times y+y
\end{array}\right\}
$$

over the signature $\mathcal{F}$ consisting of all symbols appearing in the rewrite rules. Since every normal form is of the form $\mathrm{s}^{n}(0)$ for some $n \geqslant 0$, it follows that $\mathcal{R}$ lacks external normal forms.

We start with some preliminary results.

Definition 3. Let $\mathcal{R}$ be a TRS. Two redexes $\Delta_{1}, \Delta_{2}$ are called pattern equal, denoted by $\Delta_{1} \approx \Delta_{2}$, if they have the same redex pattern, i.e., they are redexes with respect to the same rewrite rule.

Lemma 2. Let $\mathcal{R}$ be an orthogonal TRS, $\alpha \in\{\mathrm{s}, \mathrm{nv}\}$, and suppose that $\Delta \approx \Delta^{\prime}$. If $C[\Delta] \in \mathrm{WN}\left(\mathcal{R}_{\alpha}\right)$ then $C\left[\Delta^{\prime}\right] \in \mathrm{WN}\left(\mathcal{R}_{\alpha}\right)$.

Proof. Let $C[\Delta] \rightarrow^{*} t$ be a normalizing rewrite sequence in $\mathcal{R}_{\alpha}$. If we replace every descendant of $\Delta$ by $\Delta^{\prime}$ then we obtain a (possibly shorter) normalizing rewrite sequence $C\left[\Delta^{\prime}\right] \rightarrow^{*} t$. The reason is that every descendant $\Delta^{\prime \prime}$ of $\Delta$ satisfies $\Delta^{\prime \prime} \approx \Delta$ due to orthogonality and hence if $\Delta^{\prime \prime}$ is contracted to some term $u$ then $\Delta$ rewrites to the same term because the variables in the right-hand sides of the rewrite rules in $\mathcal{R}_{\alpha}$ are fresh. Moreover, as $t$ is a normal form, there are no descendants of $\Delta$ left. Note that the resulting sequence can be shorter since rewrite steps below a descendant of $\Delta$ are not mimicked.

The above lemma does not hold for the growing approximation, as shown by the following example.

Example 4. Consider the TRS $\mathcal{R}=\{\mathrm{f}(x) \rightarrow x, \mathrm{a} \rightarrow \mathrm{b}, \mathrm{c} \rightarrow \mathrm{c}\}$. We have $\mathcal{R}_{\mathrm{g}}=\mathcal{R}$. Consider the redexes $\Delta=\mathrm{f}(\mathrm{a})$ and $\Delta^{\prime}=\mathrm{f}(\mathrm{c})$. Clearly $\Delta \approx \Delta^{\prime}$. Redex $\Delta$ admits the normal form $b$, but $\Delta^{\prime}$ has no normal form.

Lemma 3. Let $\mathcal{R}$ be an orthogonal TRS over a signature $\mathcal{F}, \alpha \in\{\mathrm{s}, \mathrm{nv}\}$, and $\mathcal{F} \subseteq \mathcal{G}$. If $\mathrm{WN}\left(\mathcal{R}_{\alpha}, \mathcal{F}\right)=\mathrm{WN}\left(\mathcal{R}_{\alpha}, \mathcal{G}, \mathcal{F}\right)$ then $\mathrm{WN}_{\bullet}\left(\mathcal{R}_{\alpha}, \mathcal{F}\right)=\mathrm{WN}_{\bullet}\left(\mathcal{R}_{\alpha}, \mathcal{G}, \mathcal{F}\right)$

Proof. The inclusion $\mathrm{WN}_{\bullet}\left(\mathcal{R}_{\alpha}, \mathcal{F}\right) \subseteq \mathrm{WN}_{\bullet}\left(\mathcal{R}_{\alpha}, \mathcal{G}, \mathcal{F}\right)$ is obvious. For the reverse inclusion we reason as follows. Let $t \in \mathrm{WN}_{\bullet}\left(\mathcal{R}_{\alpha}, \mathcal{G}, \mathcal{F}\right)$ and consider a rewrite sequence $A$ in $\left(\mathcal{R}_{\alpha}, \mathcal{G}_{\bullet}\right)$ that normalizes $t$. We may write $t=C\left[t_{1}, \ldots, t_{n}\right]$ such that $t_{1}, \ldots, t_{n}$ are the maximal subterms of $t$ that are rewritten in $A$ at their root positions. Hence $A$ can be rearranged into $A^{\prime}$ :

$$
t \rightarrow_{\mathcal{R}_{\alpha}, \mathcal{G} \bullet}^{*} C\left[\Delta_{1}, \ldots, \Delta_{n}\right] \rightarrow_{\mathcal{R}_{\alpha}, \mathcal{G} \bullet}^{*} C\left[u_{1}, \ldots, u_{n}\right]
$$


for some redexes $\Delta_{1}, \ldots, \Delta_{n}$ and normal form $C\left[u_{1}, \ldots, u_{n}\right] \in \mathcal{T}(\mathcal{G})$. Since the context $C$ cannot contain $\bullet$, all occurrences of $\bullet$ are in the substitution parts of the redexes $\Delta_{1}, \ldots, \Delta_{n}$. If we replace in $C\left[\Delta_{1}, \ldots, \Delta_{n}\right]$ every $\mathcal{G} \bullet \backslash \mathcal{F}$-alien by some ground term $c \in \mathcal{T}(\mathcal{F})$, we obtain a term $t^{\prime}=C\left[\Delta_{1}^{\prime}, \ldots, \Delta_{n}^{\prime}\right]$ with $\Delta_{i}^{\prime} \in \mathcal{T}(\mathcal{F})$ and $\Delta_{i} \approx \Delta_{i}^{\prime}$ for every $i$. Repeated application of Lemma 2 yields $t^{\prime} \in \mathrm{WN}_{\bullet}\left(\mathcal{R}_{\alpha}, \mathcal{G}\right)$. Because $\bullet$ cannot contribute to the creation of a normal form, we actually have $t^{\prime} \in \mathrm{WN}\left(\mathcal{R}_{\alpha}, \mathcal{G}\right)$ and thus $t^{\prime} \in \mathrm{WN}\left(\mathcal{R}_{\alpha}, \mathcal{G}, \mathcal{F}\right)$ as $t^{\prime} \in \mathcal{T}(\mathcal{F})$. The assumption yields $t^{\prime} \in \mathrm{WN}\left(\mathcal{R}_{\alpha}, \mathcal{F}\right)$. Since $\mathrm{WN}\left(\mathcal{R}_{\alpha}, \mathcal{F}\right) \subseteq \mathrm{WN}_{\bullet}\left(\mathcal{R}_{\alpha}, \mathcal{F}\right)$ clearly holds, we obtain $t^{\prime} \in W^{\prime} \cdot\left(\mathcal{R}_{\alpha}, \mathcal{F}\right)$. Now, if we replace in the first part of $A^{\prime}$ every $\mathcal{G} \backslash \mathcal{F}$-alien by $c$ then we obtain a (possibly shorter) rewrite sequence $t \rightarrow_{\mathcal{R}_{\alpha}, \mathcal{F}_{\bullet}}^{*} C\left[\Delta_{1}^{\prime \prime}, \ldots, \Delta_{n}^{\prime \prime}\right] \in \mathcal{T}\left(\mathcal{F}_{\bullet}\right)$ with $\Delta_{i} \approx \Delta_{i}^{\prime \prime}$ and thus also $\Delta_{i}^{\prime} \approx \Delta_{i}^{\prime \prime}$ for every $i$. Repeated application of Lemma 2 yields $C\left[\Delta_{1}^{\prime \prime}, \ldots, \Delta_{n}^{\prime \prime}\right] \in \mathrm{WN}_{\bullet}\left(\mathcal{R}_{\alpha}, \mathcal{F}\right)$ and therefore $t \in \mathrm{WN}_{\mathbf{0}}\left(\mathcal{R}_{\alpha}, \mathcal{F}\right)$ as desired.

We note that for $\alpha=\mathrm{s}$ the preceding lemma is a simple consequence of Lemma 6 below. The restriction to $\alpha \in\{\mathrm{s}, \mathrm{nv}\}$ is essential. For $\mathcal{R}_{\mathrm{g}}$ we have the following counterexample.

Example 5. Consider the orthogonal TRS

$$
\mathcal{R}=\left\{\begin{array}{llll}
\mathrm{f}(x, \mathrm{a}, y) & \rightarrow \mathrm{g}(y) & \mathrm{h}(\mathrm{a}) & \rightarrow \mathrm{a} \\
\mathrm{f}(\mathrm{a}, \mathrm{b}(x), y) & \rightarrow \mathrm{a} & \mathrm{h}(\mathrm{b}(x)) & \rightarrow \mathrm{i}(x) \\
\mathrm{f}(\mathrm{b}(x), \mathrm{b}(y), z) & \rightarrow \mathrm{a} & \mathrm{i}(\mathrm{a}) & \rightarrow \mathrm{a} \\
\mathrm{g}(\mathrm{a}) & \rightarrow \mathrm{b}(\mathrm{g}(\mathrm{a})) & \mathrm{i}(\mathrm{b}(x)) & \rightarrow \mathrm{b}(\mathrm{a}) \\
\mathrm{g}(\mathrm{b}(x)) & \rightarrow \mathrm{g}(\mathrm{a}) & \mathrm{j}(x, \mathrm{a}) & \rightarrow \mathrm{f}(x, \mathrm{~h}(\mathrm{~b}(\mathrm{a})), \mathrm{a}) \\
& & \mathrm{j}(x, \mathrm{~b}(y)) \rightarrow \mathrm{f}(x, \mathrm{~h}(\mathrm{~b}(\mathrm{a})), y)
\end{array}\right\}
$$

over the signature $\mathcal{F}$ consisting of all symbols appearing in the rewrite rules and let $\mathcal{S}=\mathcal{R}_{\mathrm{g}}$. Let $\mathcal{G}=\mathcal{F} \cup\{\mathrm{c}\}$ with c a constant. With some effort one can check that $\operatorname{WN}(\mathcal{S}, \mathcal{F})=\operatorname{WN}(\mathcal{S}, \mathcal{G}, \mathcal{F})$. However, $\mathrm{WN}_{\bullet}(\mathcal{S}, \mathcal{F})$ is different from $\mathrm{WN}_{\bullet}(\mathcal{S}, \mathcal{G}, \mathcal{F})$ as witnessed by the term $t=\mathrm{j}(\bullet, \mathrm{b}(\mathrm{a}))$. In $\left(\mathcal{S}_{\bullet}, \mathcal{G}_{\bullet}\right)$ we have $t \rightarrow$ $\mathrm{f}(\bullet, \mathrm{h}(\mathrm{b}(\mathrm{a})), \mathrm{c}) \rightarrow^{+} \mathrm{f}(\bullet, \mathrm{a}, \mathrm{c}) \rightarrow \mathrm{g}(\mathrm{c})$, hence $t \in \mathrm{WN}_{\bullet}(\mathcal{S}, \mathcal{G}, \mathcal{F})$, but one easily verifies that $t$ does not have a normal form in $\left(\mathcal{S}_{\bullet}, \mathcal{F}_{\bullet}\right)$.

Lemma 4. The set of ground normal forms of a CS without external normal forms coincides with the set of ground constructor terms.

Proof. Clearly every ground constructor term is a normal form. Suppose there exists a ground normal form that contains a defined function symbol. Since every subterm of a normal form is a normal form, there must be a ground normal form $t$ whose root symbol is a defined function symbol. By definition of external normal form, $t$ must be an instance of a proper non-variable subterm of a left-hand side $l$. This implies that a proper subterm of $l$ contains a defined function symbol, contradicting the assumption that the TRS under consideration is a CS.

Lemma 5. Let $(\mathcal{R}, \mathcal{F})$ and $(\mathcal{S}, \mathcal{G})$ be orthogonal TRSs and $\alpha \in\{\mathrm{s}, \mathrm{nv}\}$ such that $(\mathcal{R}, \mathcal{F}) \subseteq(\mathcal{S}, \mathcal{G})$ and $\mathrm{WN}\left(\mathcal{S}_{\alpha}, \mathcal{G}, \mathcal{F}\right)=\mathrm{WN}\left(\mathcal{R}_{\alpha}, \mathcal{F}\right)$. If $t \in \mathrm{WNR}\left(\mathcal{S}_{\alpha}, \mathcal{G}\right)$ and $\operatorname{root}(t) \in \mathcal{F}$ then there exists a flat redex $\Xi$ in $\mathcal{T}(\mathcal{F})$. Moreover, if $\mathcal{R}_{\alpha}$ is collapsing then we may assume that $\Xi$ is $\mathcal{R}_{\alpha}$-collapsing. 
Proof. From $t \in \operatorname{WNR}\left(\mathcal{S}_{\alpha}, \mathcal{G}\right)$ we infer that $t \rightarrow_{\mathcal{S}_{\alpha}, \mathcal{G}}^{*} \Delta$ for some redex $\Delta \in$ WN $\left(\mathcal{S}_{\alpha}, \mathcal{G}\right)$. By considering the first such redex it follows that $\Delta$ is a redex with respect to $\left(\mathcal{R}_{\alpha}, \mathcal{G}\right)$. If we replace in $\Delta$ the subterms below the redex pattern by an arbitrary ground term in $\mathcal{T}(\mathcal{F})$ then we obtain a redex $\Delta^{\prime} \in \mathcal{T}(\mathcal{F})$ with $\Delta \approx \Delta^{\prime}$. Lemma 2 yields $\Delta^{\prime} \in \mathrm{WN}\left(\mathcal{S}_{\alpha}, \mathcal{G}\right)$ and thus $\Delta^{\prime} \in \mathrm{WN}\left(\mathcal{S}_{\alpha}, \mathcal{G}, \mathcal{F}\right)=\operatorname{WN}\left(\mathcal{R}_{\alpha}, \mathcal{F}\right)$. Hence $\operatorname{NF}(\mathcal{R}, \mathcal{F})=\operatorname{NF}\left(\mathcal{R}_{\alpha}, \mathcal{F}\right) \neq \varnothing$. Therefore, using orthogonality, we obtain a flat redex $\Xi \in \mathcal{T}(\mathcal{F})$ by replacing the variables in the left-hand side of any rewrite rule in $\mathcal{R}$ by terms in $\operatorname{NF}(\mathcal{R}, \mathcal{F})$. If $\mathcal{R}_{\alpha}$ is a collapsing then we take any $\mathcal{R}_{\alpha}$-collapsing rewrite rule.

We are now ready for the final signature extension result of this section. The condition $\operatorname{WN}\left(\mathcal{R}_{\alpha}, \mathcal{F}\right)=\operatorname{WN}\left(\mathcal{R}_{\alpha}, \mathcal{G}, \mathcal{F}\right)$ expresses that the set of $\mathcal{R}_{\alpha^{-}}$ normalizable terms in $\mathcal{T}(\mathcal{F})$ is not enlarged by allowing terms in $\mathcal{T}(\mathcal{G})$ to be substituted for the variables in the rewrite rules. We stress that this condition is decidable for left-linear $\mathcal{R}$ and $\alpha \in\{\mathrm{s}, \mathrm{nv}, \mathrm{g}\}$ by standard tree automata techniques.

Theorem 3. Let $\mathcal{R}$ be an orthogonal TRS over a signature $\mathcal{F}, \alpha \in\{\mathrm{s}, \mathrm{nv}\}$, and $\mathcal{F} \subseteq \mathcal{G}$ such that $\operatorname{WN}\left(\mathcal{R}_{\alpha}, \mathcal{F}\right)=\operatorname{WN}\left(\mathcal{R}_{\alpha}, \mathcal{G}, \mathcal{F}\right)$. If $(\mathcal{R}, \mathcal{F}) \in \mathcal{C B N} \mathcal{N}_{\alpha}$ and $\mathcal{R}$ is a CS or $\mathcal{R}_{\alpha}$ is collapsing then $(\mathcal{R}, \mathcal{G}) \in \mathcal{C B N}_{\alpha}$.

Proof. If $(\mathcal{R}, \mathcal{F})$ has external normal forms then the result follows from Theorem [2. So we assume that $(\mathcal{R}, \mathcal{F})$ lacks external normal forms. We also assume that $\mathcal{R} \neq \varnothing$ for otherwise the result is trivial. Suppose to the contrary that $(\mathcal{R}, \mathcal{G}) \notin \mathcal{C B N}_{\alpha}$. According to Lemma 1 there exists a term $t \in \operatorname{WNR}\left(\mathcal{R}_{\alpha}, \mathcal{G}\right)$ without $\left(\mathcal{R}_{\alpha}, \mathcal{G}\right)$-needed redex. Lemma 5 (with $\mathcal{R}=\mathcal{S}$ ) yields a flat redex $\Xi \in \mathcal{T}(\mathcal{F})$. If $\mathcal{R}_{\alpha}$ is collapsing then we may assume that $\Xi$ is $\mathcal{R}_{\alpha}$-collapsing. Let $t^{\prime}$ be the term in $\mathcal{T}(\mathcal{F})$ obtained from $t$ by replacing every $\mathcal{G} \backslash \mathcal{F}$-alien by $\Xi$. Let $P$ be the set of positions of those aliens. Since $t^{\prime}$ is reducible, it contains an $\left(\mathcal{R}_{\alpha}, \mathcal{F}\right)$-needed redex, say at position $q$. We show that $t^{\prime}[\bullet]_{q} \in \mathrm{WN}_{\bullet}\left(\mathcal{R}_{\alpha}, \mathcal{G}\right)$. We consider two cases.

1. Suppose that $q \in P$. Since $t \in \operatorname{WNR}\left(\mathcal{R}_{\alpha}, \mathcal{G}\right), t \rightarrow_{\mathcal{R}_{\alpha}, \mathcal{G}}^{*} \Delta$ for some redex $\Delta \in \mathrm{WN}\left(\mathcal{R}_{\alpha}, \mathcal{G}\right) \subseteq \mathrm{WN}_{\bullet}\left(\mathcal{R}_{\alpha}, \mathcal{G}\right)$. Since the root symbol of every alien belongs to $\mathcal{G} \backslash \mathcal{F}$, aliens cannot contribute to the creation of $\Delta$ and hence we may replace them by arbitrary terms in $\mathcal{T}\left(\mathcal{G}_{\bullet}\right)$ and still obtain a redex that is pattern equal to $\Delta$. We replace the alien at position $q$ by $\bullet$ and every alien at position $p \in P \backslash\{q\}$ by $\left.t^{\prime}\right|_{p}$. This gives $t^{\prime}[\bullet]_{q} \rightarrow_{\mathcal{R}_{\alpha}, \mathcal{G}_{\bullet}}^{*} \Delta^{\prime}$ with $\Delta^{\prime} \approx \Delta$. Lemma 2 yields $\Delta^{\prime} \in \mathrm{WN}_{\bullet}\left(\mathcal{R}_{\alpha}, \mathcal{G}\right)$ and hence $t^{\prime}[\bullet]_{q} \in \mathrm{WN}_{\bullet}\left(\mathcal{R}_{\alpha}, \mathcal{G}\right)$.

2. Suppose that $q \notin P$. Since $\Xi$ is flat, it follows by orthogonality that $q$ is also a redex position in $t$. Since $t$ is an $\left(\mathcal{R}_{\alpha}, \mathcal{G}\right)$-free term, $t[\bullet]_{q} \in \mathrm{WN}_{\bullet}\left(\mathcal{R}_{\alpha}, \mathcal{G}\right)$. We distinguish two further cases.

a) First assume that $\mathcal{R}$ is a CS. Since $t$ is not root-stable, its root symbol must be defined. From Lemma 4 we learn that a ground normal form of $\mathcal{R}$ (and thus of $\mathcal{R}_{\alpha}$ ) cannot contain defined symbols. Hence any $\left(\mathcal{R}_{\alpha}, \mathcal{G}_{\bullet}\right)$ rewrite sequence that normalizes $t[\bullet]_{q}$ contains a root step and thus $t[\bullet]_{q} \in \mathrm{WNR}_{\bullet}\left(\mathcal{R}_{\alpha}, \mathcal{G}\right)$. Hence $t[\bullet]_{q} \rightarrow_{\mathcal{R}_{\alpha}, \mathcal{G} \bullet}^{*} \Delta^{\prime} \in \mathrm{WN}_{\bullet}\left(\mathcal{R}_{\alpha}, \mathcal{G}\right)$ for some 
redex $\Delta^{\prime} \in \mathcal{T}\left(\mathcal{G}_{\bullet}\right)$. Similar to case (1) above, we replace the $\mathcal{G} \backslash \mathcal{F}$-aliens in $t[\bullet]_{q}$ by $\Xi$. This yields $t^{\prime}[\bullet]_{q} \rightarrow_{\mathcal{R}_{\alpha}, \mathcal{G}_{\bullet}}^{*} \Delta^{\prime \prime}$ with $\Delta^{\prime \prime} \approx \Delta^{\prime}$. Lemma 2 yields $\Delta^{\prime \prime} \in \mathrm{WN}_{\bullet}\left(\mathcal{R}_{\alpha}, \mathcal{G}\right)$ and hence $t^{\prime}[\bullet]_{q} \in \mathrm{WN}_{\bullet}\left(\mathcal{R}_{\alpha}, \mathcal{G}\right)$.

b) Next assume that $\mathcal{R}_{\alpha}$ is collapsing. Because $\Xi$ is a collapsing redex, we have $\left.\Xi \rightarrow_{\mathcal{R}_{\alpha}, \mathcal{G}} t\right|_{p}$ for all $p \in P$. Hence $t^{\prime}[\bullet]_{q} \rightarrow_{\mathcal{R}_{\alpha}, \mathcal{G} \bullet}^{*} t[\bullet]_{q}$ and thus $t^{\prime}[\bullet]_{q} \in \mathrm{WN}_{\bullet}\left(\mathcal{R}_{\alpha}, \mathcal{G}\right)$.

As $t^{\prime} \in \mathcal{T}(\mathcal{F})$, we have $t^{\prime}[\bullet]_{q} \in \mathrm{WN}_{\bullet}\left(\mathcal{R}_{\alpha}, \mathcal{G}, \mathcal{F}\right)$ and thus $t^{\prime}[\bullet]_{q} \notin \mathrm{WN}_{\bullet}\left(\mathcal{R}_{\alpha}, \mathcal{F}\right)$ by Lemma 3 contradicting the assumption that $q$ is the position of an $\left(\mathcal{R}_{\alpha}, \mathcal{F}\right)$ needed redex in $t^{\prime}$.

It can be shown that all conditions in the above theorem are necessary. Below we show the necessity of the $\operatorname{WN}\left(\mathcal{R}_{\alpha}, \mathcal{F}\right)=\mathrm{WN}\left(\mathcal{R}_{\alpha}, \mathcal{G}, \mathcal{F}\right)$ condition for noncollapsing CSs $\mathcal{R}_{\alpha}$. Due to lack of space we omit the other (complicated) counterexamples.

Example 6. Consider the orthogonal noncollapsing CS

$$
\mathcal{R}=\left\{\begin{array}{ll}
\mathrm{f}(x, \mathrm{a}, \mathrm{b}) \rightarrow \mathrm{g}(x) & \mathrm{g}(\mathrm{a}) \rightarrow \mathrm{g}(\mathrm{a}) \\
\mathrm{f}(\mathrm{b}, x, \mathrm{a}) \rightarrow \mathrm{g}(x) & \mathrm{g}(\mathrm{b}) \rightarrow \mathrm{g}(\mathrm{a}) \\
\mathrm{f}(\mathrm{a}, \mathrm{b}, x) \rightarrow \mathrm{g}(x) & \mathrm{h}(x) \rightarrow \mathrm{i}(x) \\
\mathrm{f}(\mathrm{a}, \mathrm{a}, \mathrm{a}) \rightarrow \mathrm{a} & \mathrm{i}(\mathrm{a}) \rightarrow \mathrm{a} \\
\mathrm{f}(\mathrm{b}, \mathrm{b}, \mathrm{b}) \rightarrow \mathrm{a} & \mathrm{i}(\mathrm{b}) \rightarrow \mathrm{b}
\end{array}\right\}
$$

over the signature $\mathcal{F}$ consisting of all symbols appearing in the rewrite rules and let $\mathcal{S}=\mathcal{R}_{\mathrm{nv}}$. Let $\mathcal{G}=\mathcal{F} \cup\{\mathrm{c}\}$ with c a constant. Note that $\operatorname{WN}(\mathcal{S}, \mathcal{F}) \neq$ $\operatorname{WN}(\mathcal{S}, \mathcal{G}, \mathcal{F})$ as witnessed by the term $\mathrm{f}(\mathrm{a}, \mathrm{a}, \mathrm{b})$. With some effort one can check that $(\mathcal{R}, \mathcal{F}) \in \mathcal{C} \mathcal{B N}$. However, $(\mathcal{R}, \mathcal{G}) \notin \mathcal{C B N}$ as $\mathrm{f}(\mathrm{h}(\mathrm{a}), \mathrm{h}(\mathrm{a}), \mathrm{h}(\mathrm{a}))$ lacks $(\mathcal{S}, \mathcal{G})$ needed redexes.

We show that Theorem 1 is a special case of Theorem 3 by proving that for $\alpha=$ s the condition $\operatorname{WN}\left(\mathcal{R}_{\alpha}, \mathcal{F}\right)=\operatorname{WN}\left(\mathcal{R}_{\alpha}, \mathcal{G}, \mathcal{F}\right)$ is a consequence of $\operatorname{NF}(\mathcal{R}, \mathcal{F}) \neq \varnothing$.

Lemma 6. Let $\mathcal{R}$ be an eTRS over a signature $\mathcal{F}$. If $\operatorname{NF}(\mathcal{R}, \mathcal{F}) \neq \varnothing$ then $\mathrm{WN}\left(\mathcal{R}_{\mathrm{s}}, \mathcal{F}\right)=\mathcal{T}(\mathcal{F})$.

Proof. If $\operatorname{NF}(\mathcal{R}, \mathcal{F}) \neq \varnothing$ then there must be a constant $c \in \operatorname{NF}(\mathcal{R}, \mathcal{F})$. Define the TRS $\mathcal{R}^{\prime}=\{l \rightarrow c \mid l \rightarrow r \in \mathcal{R}\}$ over the signature $\mathcal{F}$. Clearly $\rightarrow_{\mathcal{R}^{\prime}} \subseteq \rightarrow_{\mathrm{s}}$. Consider a precedence (i.e., a well-founded proper order on $\mathcal{F}$ ) $>$ with $f>c$ for every function symbol $f \in \mathcal{F}$ different from $c$. The TRS $\mathcal{R}^{\prime}$ is compatible with the induced recursive path order $>_{\text {rpo }}([3])$ and thus terminating. Since $\mathcal{R}_{\mathrm{s}}$ and $\mathcal{R}^{\prime}$ have the same normal forms, it follows that $\mathcal{R}_{\mathrm{s}}$ is weakly normalizing.

Proof of Theorem 1 Let $\mathcal{R}$ be an orthogonal TRS over a signature $\mathcal{F}$ such that $(\mathcal{R}, \mathcal{F}) \in \mathcal{C B} \mathcal{N}_{\mathrm{s}}$. Let $\mathcal{F} \subseteq \mathcal{G}$. We have to show that $(\mathcal{R}, \mathcal{G}) \in \mathcal{C} \mathcal{B N}_{\mathrm{s}}$. Since $\mathcal{R}_{\mathrm{s}}$ is collapsing (if $\mathcal{R} \neq \varnothing$; otherwise $\mathcal{R}$ is a CS), the result follows from Theorem 3 provided that $\mathrm{WN}\left(\mathcal{R}_{\mathrm{s}}, \mathcal{F}\right)=\mathrm{WN}\left(\mathcal{R}_{\mathrm{s}}, \mathcal{G}, \mathcal{F}\right)$. From Lemma 6 we obtain $\operatorname{WN}\left(\mathcal{R}_{\mathrm{s}}, \mathcal{F}\right)=\mathcal{T}(\mathcal{F})$ and $\operatorname{WN}\left(\mathcal{R}_{\mathrm{s}}, \mathcal{G}, \mathcal{F}\right)=\mathrm{WN}\left(\mathcal{R}_{\mathrm{s}}, \mathcal{G}\right) \cap \mathcal{T}(\mathcal{F})=\mathcal{T}(\mathcal{G}) \cap \mathcal{T}(\mathcal{F})=$ $\mathcal{T}(\mathcal{F})$. 
We conclude this section by remarking that we have to use Theorem 3 only once. After adding a single new function symbol we obtain an external normal form and hence we can apply Theorem 2 for the remaining new function symbols.

\section{Modularity}

The results obtained in the previous section form the basis for the modularity results presented in this section. We first consider disjoint combinations.

Definition 4. We say that a class $\mathcal{C}$ of TRSs is modular (for disjoint combinations) if $\left(\mathcal{R} \cup \mathcal{R}^{\prime}, \mathcal{F} \cup \mathcal{F}^{\prime}\right) \in \mathcal{C}$ for all $(\mathcal{R}, \mathcal{F}),\left(\mathcal{R}^{\prime}, \mathcal{F}^{\prime}\right) \in \mathcal{C}$ such that $\mathcal{F} \cap \mathcal{F}^{\prime}=\varnothing$.

To simplify notation, in the remainder of this section we write $\mathcal{S}$ for $\mathcal{R} \cup \mathcal{R}^{\prime}$ and $\mathcal{G}$ for $\mathcal{F} \cup \mathcal{F}^{\prime}$. The condition in Theorem 2 is insufficient for modularity as shown by the following example.

Example 7. Consider the TRS

$$
\mathcal{R}=\left\{\begin{array}{l}
\mathrm{f}(x, \mathrm{a}, \mathrm{b}) \rightarrow \mathrm{a} \\
\mathrm{f}(\mathrm{b}, x, \mathrm{a}) \rightarrow \mathrm{a} \\
\mathrm{f}(\mathrm{a}, \mathrm{b}, x) \rightarrow \mathrm{a}
\end{array}\right\}
$$

over the signature $\mathcal{F}$ consisting of all symbols appearing in the rewrite rules and the TRS $\mathcal{R}^{\prime}=\{\mathrm{g}(x) \rightarrow x\}$ over the signature $\mathcal{F}^{\prime}$ consisting of a constant $\mathrm{c}$ in addition to $\mathrm{g}$. Both TRSs have external normal forms and belong to $\mathcal{C} \mathcal{B N}_{\text {nv }}$, as one easily shows. Their union does not belong to $\mathcal{C} \mathcal{B N}_{\text {nv }}$ as the term $\mathrm{f}(\mathrm{g}(\mathrm{a}), \mathrm{g}(\mathrm{a}), \mathrm{g}(\mathrm{a}))$ has no $\left(\mathcal{S}_{\text {nv }}, \mathcal{G}\right)$-needed redex:

$$
\begin{aligned}
& \mathrm{f}(\bullet, \mathrm{g}(\mathrm{a}), \mathrm{g}(\mathrm{a})) \rightarrow_{\mathrm{nv}} \mathrm{f}(\bullet, \mathrm{a}, \mathrm{g}(\mathrm{a})) \rightarrow_{\mathrm{nv}} \mathrm{f}(\bullet, \mathrm{a}, \mathrm{b}) \rightarrow_{\mathrm{nv}} \mathrm{a} \\
& \mathrm{f}(\mathrm{g}(\mathrm{a}), \bullet, \mathrm{g}(\mathrm{a})) \rightarrow_{\mathrm{nv}} \mathrm{f}(\mathrm{b}, \bullet, \mathrm{g}(\mathrm{a})) \rightarrow_{\mathrm{nv}} \mathrm{f}(\mathrm{b}, \bullet, \mathrm{a}) \rightarrow_{\mathrm{nv}} \mathrm{a} \\
& \mathrm{f}(\mathrm{g}(\mathrm{a}), \mathrm{g}(\mathrm{a}), \bullet) \rightarrow_{\mathrm{nv}} \mathrm{f}(\mathrm{a}, \mathrm{g}(\mathrm{a}), \bullet) \rightarrow_{\mathrm{nv}} \mathrm{f}(\mathrm{a}, \mathrm{b}, \bullet) \rightarrow_{\mathrm{nv}} \mathrm{a}
\end{aligned}
$$

If we forbid collapsing rules like $\mathrm{g}(x) \rightarrow x$, modularity holds. The following theorem can be proved along the lines of the proof of Theorem 2 because there are no collapsing rules and the eTRSs are left-linear, aliens cannot influence the possibility to perform a rewrite step in the non-alien part of a term.

Theorem 4. The subclass of $\mathcal{C B N}$ consisting of all noncollapsing left-linear eTRSs with external normal forms is modular.

We find it convenient to separate the counterpart of Theorem 3 into two parts. The next two lemmata are used in both proofs. The nontrivial proof of the first one is omitted due to lack of space.

Lemma 7. Let $(\mathcal{R}, \mathcal{F})$ and $\left(\mathcal{R}^{\prime}, \mathcal{F}^{\prime}\right)$ be disjoint TRSs. If $\alpha \in\{\mathrm{s}, \mathrm{nv}\}$ then $\mathrm{WN}\left(\mathcal{S}_{\alpha}, \mathcal{G}, \mathcal{F}\right)=\operatorname{WN}\left(\mathcal{R}_{\alpha}, \mathcal{G}, \mathcal{F}\right)$.

Lemma 8. Let $(\mathcal{R}, \mathcal{F})$ and $\left(\mathcal{R}^{\prime}, \mathcal{F}^{\prime}\right)$ be disjoint orthogonal TRSs and $\alpha \in$ $\{\mathrm{s}, \mathrm{nv}\}$. If $\operatorname{WN}\left(\mathcal{S}_{\alpha}, \mathcal{G}, \mathcal{F}\right)=\operatorname{WN}\left(\mathcal{R}_{\alpha}, \mathcal{F}\right)$ then $\mathrm{WN}_{\bullet}\left(\mathcal{S}_{\alpha}, \mathcal{G}, \mathcal{F}\right)=\mathrm{WN}_{\bullet}\left(\mathcal{R}_{\alpha}, \mathcal{F}\right)$. 
Proof. The previous lemma yields $\mathrm{WN}\left(\mathcal{R}_{\alpha}, \mathcal{F}\right)=\mathrm{WN}\left(\mathcal{R}_{\alpha}, \mathcal{G}, \mathcal{F}\right)$. From Lemma 3 we obtain $\mathrm{WN}_{\bullet}\left(\mathcal{R}_{\alpha}, \mathcal{F}\right)=\mathrm{WN}_{\bullet}\left(\mathcal{R}_{\alpha}, \mathcal{G}, \mathcal{F}\right)$. Another application of the previous lemma yields the desired $\mathrm{WN}_{\bullet}\left(\mathcal{R}_{\alpha}, \mathcal{F}\right)=\mathrm{WN}_{\bullet}\left(\mathcal{S}_{\alpha}, \mathcal{G}, \mathcal{F}\right)$.

Theorem 5. Let $(\mathcal{R}, \mathcal{F})$ and $\left(\mathcal{R}^{\prime}, \mathcal{F}^{\prime}\right)$ be disjoint orthogonal noncollapsing CSs such that $\mathrm{WN}\left(\mathcal{R}_{\mathrm{nv}}, \mathcal{G}, \mathcal{F}\right)=\mathrm{WN}\left(\mathcal{R}_{\mathrm{nv}}, \mathcal{F}\right)$ and $\mathrm{WN}\left(\mathcal{R}_{\mathrm{nv}}^{\prime}, \mathcal{G}, \mathcal{F}^{\prime}\right)=\mathrm{WN}\left(\mathcal{R}_{\mathrm{nv}}^{\prime}, \mathcal{F}^{\prime}\right)$. If $(\mathcal{R}, \mathcal{F}),\left(\mathcal{R}^{\prime}, \mathcal{F}^{\prime}\right) \in \mathcal{C} \mathcal{B} \mathcal{N}_{\mathrm{nv}}$ then $(\mathcal{S}, \mathcal{G}) \in \mathcal{C B N}_{\mathrm{nv}}$.

Proof. We assume that both $\mathcal{R}$ and $\mathcal{R}^{\prime}$ are nonempty, for otherwise the result follows from Theorem 3. Suppose to the contrary that $(\mathcal{S}, \mathcal{G}) \notin \mathcal{C B} \mathcal{N}_{\text {nv }}$. According to Lemma 1 there exists a term $t \in \operatorname{WNR}\left(\mathcal{S}_{\text {nv }}, \mathcal{G}\right)$ without $\left(\mathcal{S}_{\text {nv }}, \mathcal{G}\right)$-needed redex. Assume without loss of generality that $\operatorname{root}(t) \in \mathcal{F}$. Lemma 5 yields a flat redex $\Xi \in \mathcal{T}(\mathcal{F})$. Let $t^{\prime}$ be the term in $\mathcal{T}(\mathcal{F})$ obtained from $t$ by replacing every $\mathcal{G} \backslash \mathcal{F}$-alien by $\Xi$. Let $P$ be the set of positions of those aliens. Since $t^{\prime}$ is reducible, it contains an $\left(\mathcal{R}_{\mathrm{nv}}, \mathcal{F}\right)$-needed redex, say at position $q$. We show that $t^{\prime}[\bullet]_{q} \in \mathrm{WN}_{\bullet}\left(\mathcal{S}_{\mathrm{nv}}, \mathcal{G}\right)$. We consider two cases.

1. Suppose that $q \in P$. Since $t \in \operatorname{WNR}\left(\mathcal{S}_{\mathrm{nv}}, \mathcal{G}\right), t \rightarrow_{\mathcal{S}_{\mathrm{nv}}, \mathcal{G}}^{*} \Delta$ for some redex $\Delta \in$ $\mathrm{WN}\left(\mathcal{S}_{\mathrm{nv}}, \mathcal{G}\right) \subseteq \mathrm{WN}_{\bullet}\left(\mathcal{S}_{\mathrm{nv}}, \mathcal{G}\right)$. Since $\mathcal{S}_{\mathrm{nv}}$ is noncollapsing and the root symbol of every alien belongs to $\mathcal{G} \backslash \mathcal{F}$, aliens cannot contribute to the creation of $\Delta$ and hence we may replace them by arbitrary terms in $\mathcal{T}\left(\mathcal{G}_{\bullet}\right)$ and still obtain a redex that is pattern equal to $\Delta$. We replace the alien at position $q$ by $\bullet$ and every alien at position $p \in P \backslash\{q\}$ by $\left.t^{\prime}\right|_{p}$. This gives $t^{\prime}[\bullet]_{q} \rightarrow_{\mathcal{S}_{\mathrm{nv}}}^{*}, \mathcal{G}_{\bullet} \Delta^{\prime}$ with $\Delta^{\prime} \approx \Delta$. Lemma 2 yields $\Delta^{\prime} \in \mathrm{WN}_{\bullet}\left(\mathcal{S}_{\mathrm{nv}}, \mathcal{G}\right)$ and hence $t^{\prime}[\bullet]_{q} \in \mathrm{WN}_{\bullet}\left(\mathcal{S}_{\mathrm{nv}}, \mathcal{G}\right)$.

2. Suppose that $q \notin P$. Since $\Xi$ is flat, it follows by orthogonality that $q$ is also a redex position in $t$. Since $t$ is an $\left(\mathcal{S}_{\mathrm{nv}}, \mathcal{G}\right)$-free term, $t[\bullet]_{q} \in \mathrm{WN}_{\bullet}\left(\mathcal{S}_{\mathrm{nv}}, \mathcal{G}\right)$. Since $t$ is not root-stable, its root symbol must be defined. From Lemma 4 we learn that a ground normal form of $\mathcal{S}$ (and thus of $\mathcal{S}_{\text {nv }}$ ) cannot contain defined symbols. Hence any $\left(\mathcal{S}_{\text {nv }}, \mathcal{G}_{\bullet}\right)$-rewrite sequence that normalizes $t[\bullet]_{q}$ contains a root step and thus $t[\bullet]_{q} \in \operatorname{WNR}_{\bullet}\left(\mathcal{S}_{\mathrm{nv}}, \mathcal{G}\right)$. Hence $t[\bullet]_{q} \rightarrow_{\mathcal{S}_{\mathrm{nv}}}^{*}, \mathcal{G}_{\bullet} \Delta \in$ $\mathrm{WN}_{\bullet}\left(\mathcal{S}_{\mathrm{nv}}, \mathcal{G}\right)$ for some redex $\Delta \in \mathcal{T}\left(\mathcal{G}_{\bullet}\right)$. We replace the $\mathcal{G} \backslash \mathcal{F}$-aliens in $t[\bullet]_{q}$ by $\Xi$. Since $\mathcal{S}$ is noncollapsing, this yields $t^{\prime}[\bullet]_{q} \rightarrow_{\mathcal{S}_{\text {nv }}, \mathcal{G}_{\bullet}}^{*} \Delta^{\prime}$ with $\Delta^{\prime} \approx \Delta$. Lemma 2 yields $\Delta^{\prime} \in \mathrm{WN}_{\bullet}\left(\mathcal{S}_{\mathrm{nv}}, \mathcal{G}\right)$ and hence $t^{\prime}[\bullet]_{q} \in \mathrm{WN}_{\bullet}\left(\mathcal{S}_{\mathrm{nv}}, \mathcal{G}\right)$.

As $t^{\prime} \in \mathcal{T}(\mathcal{F})$, we have $t^{\prime}[\bullet]_{q} \in \mathrm{WN}_{\bullet}\left(\mathcal{S}_{\mathrm{nv}}, \mathcal{G}, \mathcal{F}\right)$ and thus $t^{\prime}[\bullet]_{q} \in \mathrm{WN}_{\bullet}\left(\mathcal{R}_{\mathrm{nv}}, \mathcal{F}\right)$ by Lemmata $[7$ and $[8$, contradicting the assumption that $q$ is the position of an $\left(\mathcal{R}_{\text {nv }}, \mathcal{F}\right)$-needed redex in $t^{\prime}$

Theorem 6. Let $(\mathcal{R}, \mathcal{F})$ and $\left(\mathcal{R}^{\prime}, \mathcal{F}^{\prime}\right)$ be disjoint orthogonal TRSs and $\alpha \in$ $\{\mathrm{s}, \mathrm{nv}\}$ such that $\operatorname{WN}\left(\mathcal{R}_{\alpha}, \mathcal{G}, \mathcal{F}\right)=\operatorname{WN}\left(\mathcal{R}_{\alpha}, \mathcal{F}\right)$ and $\operatorname{WN}\left(\mathcal{R}_{\alpha}^{\prime}, \mathcal{G}, \mathcal{F}^{\prime}\right)=$ $\operatorname{WN}\left(\mathcal{R}_{\alpha}^{\prime}, \mathcal{F}^{\prime}\right)$. If $(\mathcal{R}, \mathcal{F}),\left(\mathcal{R}^{\prime}, \mathcal{F}^{\prime}\right) \in \mathcal{C B N}_{\alpha}$ and both $\mathcal{R}_{\alpha}$ and $\mathcal{R}_{\alpha}^{\prime}$ are collapsing then $(\mathcal{S}, \mathcal{G}) \in \mathcal{C B N}_{\alpha}$.

Proof. We assume that both $\mathcal{R}$ and $\mathcal{R}^{\prime}$ are nonempty, for otherwise the result follows from Theorem 3. Suppose to the contrary that $(\mathcal{S}, \mathcal{G}) \notin \mathcal{C} \mathcal{B N}_{\alpha}$. According to Lemma 1 there exists a term $t \in \operatorname{WNR}\left(\mathcal{S}_{\alpha}, \mathcal{G}\right)$ without $\left(\mathcal{S}_{\alpha}, \mathcal{G}\right)$-needed redex. Assume without loss of generality that $\operatorname{root}(t) \in \mathcal{F}^{\prime}$. Lemma 5 yields a 
flat $\mathcal{R}_{\alpha}^{\prime}$-collapsing redex $\Xi \in \mathcal{T}\left(\mathcal{F}^{\prime}\right)$. Let $t^{\prime}$ be the term in $\mathcal{T}\left(\mathcal{F}^{\prime}\right)$ obtained from $t$ by replacing every $\mathcal{G} \backslash \mathcal{F}^{\prime}$-alien by $\Xi$. Let $P$ be the set of positions of those aliens. Since $t^{\prime}$ is reducible, it contains an $\left(\mathcal{R}_{\alpha}^{\prime}, \mathcal{F}^{\prime}\right)$-needed redex, say at position $q$. We show that $t^{\prime}[\bullet]_{q} \in \mathrm{WN} \bullet\left(\mathcal{S}_{\alpha}, \mathcal{G}\right)$. Because $\Xi$ is a collapsing redex, we have $\left.\Xi \rightarrow_{\mathcal{R}_{\alpha}, \mathcal{G}} t\right|_{p}$ for all $p \in P$. Hence $t^{\prime} \rightarrow_{\mathcal{R}_{\alpha}, \mathcal{G}_{\bullet}}^{*} t$ and thus, by orthogonality, $t^{\prime}[\bullet]_{q} \rightarrow_{\mathcal{R}_{\alpha}, \mathcal{G}_{\bullet}}^{*} t[\bullet]_{q}$. Hence it suffices to show that $t[\bullet]_{q} \in \mathrm{WN}_{\bullet}\left(\mathcal{S}_{\alpha}, \mathcal{G}\right)$. We distinguish two cases.

1. Suppose that $q \in P$. Since $t \in \operatorname{WNR}\left(\mathcal{S}_{\alpha}, \mathcal{G}\right), t \rightarrow \rightarrow_{\mathcal{S}_{\alpha}, \mathcal{G}}^{*} \Delta$ for some redex $\Delta \in \mathrm{WN}\left(\mathcal{S}_{\alpha}, \mathcal{G}\right) \subseteq \mathrm{WN}_{\bullet}\left(\mathcal{S}_{\alpha}, \mathcal{G}\right)$. We distinguish two further cases.

a) If $\left.t\right|_{q}$ is a normal form then it cannot contribute to the creation of $\Delta$ and hence by replacing it by $\bullet$ we obtain $t[\bullet]_{q} \rightarrow_{\mathcal{S}_{\alpha}, \mathcal{G}}^{*} \Delta^{\prime}$ with $\Delta \approx \Delta^{\prime}$. Lemma 2 yields $\Delta^{\prime} \in \mathrm{WN}_{\bullet}\left(\mathcal{S}_{\alpha}, \mathcal{G}\right)$ and thus $t[\bullet]_{q} \in \mathrm{WN}_{\bullet}\left(\mathcal{S}_{\alpha}, \mathcal{G}\right)$.

b) Suppose $\left.t\right|_{q}$ is reducible. Because $t$ is a minimal free term, $\left.t\right|_{q}$ contains an $\left(\mathcal{S}_{\alpha}, \mathcal{G}\right)$-needed redex, say at position $q^{\prime}$. So $\left.t\right|_{q}[\bullet]_{q^{\prime}} \notin \mathrm{WN}_{\bullet}\left(\mathcal{S}_{\alpha}, \mathcal{G}\right)$. In particular, $\left.t\right|_{q}[\bullet]_{q^{\prime}}$ does not $\left(\mathcal{S}_{\alpha}, \mathcal{G}\right)$-rewrite to a collapsing redex, for otherwise it would rewrite to a normal form in one extra step. Hence the root symbol of every reduct of $\left.t\right|_{q}[\bullet]_{q^{\prime}}$ belongs to $\mathcal{F}$. Since $q q^{\prime}$ is not the position of an $\left(\mathcal{S}_{\alpha}, \mathcal{G}\right)$-needed redex in $t, t[\bullet]_{q q^{\prime}} \in \mathrm{WN}_{\bullet}\left(\mathcal{S}_{\alpha}, \mathcal{G}\right)$. Since any normalizing $\left(\mathcal{S}_{\alpha}, \mathcal{G}\right)$-rewrite sequence must contain a rewrite step at a position above $q$, we may write $t[\bullet]_{q q^{\prime}} \rightarrow_{\mathcal{S}_{\alpha}, \mathcal{G}}^{*} C\left[\Delta^{\prime}\right] \in \mathrm{WN}_{\bullet}\left(\mathcal{S}_{\alpha}, \mathcal{G}\right)$ such that $\Delta^{\prime}$ is the first redex above position $q$. Since $\operatorname{root}\left(\Delta^{\prime}\right) \in \mathcal{F}^{\prime}$, the subterm $\left.t\right|_{q}[\bullet]_{q^{\prime}}$ of $t[\bullet]_{q q^{\prime}}$ does not contribute to the creation of $\Delta^{\prime}$ and hence $t[\bullet]_{q} \rightarrow_{\mathcal{S}_{\alpha}, \mathcal{G}}^{*} C\left[\Delta^{\prime \prime}\right]$ with $\Delta^{\prime \prime} \approx \Delta^{\prime}$. Lemma 2 yields $C\left[\Delta^{\prime \prime}\right] \in$ $\mathrm{WN}_{\bullet}\left(\mathcal{S}_{\alpha}, \mathcal{G}\right)$ and thus $t[\bullet]_{q} \in \mathrm{WN}_{\bullet}\left(\mathcal{S}_{\alpha}, \mathcal{G}\right)$.

2. Suppose that $q \notin P$. Since $\Xi$ is flat, it follows by orthogonality that $q$ is also a redex position in $t$. Since $t$ is an $\left(\mathcal{S}_{\alpha}, \mathcal{G}\right)$-free term, $t[\bullet]_{q} \in \mathrm{WN}_{\bullet}\left(\mathcal{S}_{\alpha}, \mathcal{G}\right)$.

As $t^{\prime} \in \mathcal{T}\left(\mathcal{F}^{\prime}\right)$, we have $t^{\prime}[\bullet]_{q} \in \mathrm{WN}_{\bullet}\left(\mathcal{S}_{\alpha}, \mathcal{G}, \mathcal{F}^{\prime}\right)$ and thus $t^{\prime}[\bullet]_{q} \in \mathrm{WN}_{\bullet}\left(\mathcal{R}_{\alpha}^{\prime}, \mathcal{F}^{\prime}\right)$ by Lemmata 7 and 8 , contradicting the assumption that $q$ is the position of an $\left(\mathcal{R}_{\alpha}^{\prime}, \mathcal{F}^{\prime}\right)$-needed redex in $t^{\prime}$.

It is rather surprising that the presence of collapsing rules helps to achieve modularity; for most properties of TRSs collapsing rules are an obstacle for modularity (see e.g. Middeldorp [14]).

The next result is the modularity counterpart of Theorem 11. It is an easy corollary of the preceding theorem.

Theorem 7. The subclass of $\mathcal{C B N}_{\mathrm{s}}$ consisting of all orthogonal TRSs $(\mathcal{R}, \mathcal{F})$ such that $\operatorname{NF}(\mathcal{R}, \mathcal{F}) \neq \varnothing$ is modular.

Klop and Middeldorp 13. showed the related result that strong sequentiality is a modular property of orthogonal TRSs. We already remarked in the preceding section that $\mathcal{C B N}_{\mathrm{s}}$ properly includes all strongly sequential TRSs. Actually, in [13] it is remarked that it is sufficient that the left-hand sides of the two strongly sequential rewrite systems do not share function symbols. One easily verifies that for our modularity results it is sufficient that $\mathcal{R}_{\alpha}$ and $\mathcal{R}_{\alpha}^{\prime}$ do not share function symbols. Actually, we can go a step further by considering so-called 
constructor-sharing combinations. In such combinations the participating systems may share constructors but not defined symbols. It can be shown that the results obtained in this section extend to constructor-sharing combinations, provided we strengthen the requirements in Theorems 4, 5, and 6] by forbidding the presence of constructor-lifting rules. A rewrite rule $l \rightarrow r$ is called constructorlifting if $\operatorname{root}(r)$ is a shared constructor. The necessity of this condition is shown in the following examples.

Example 8. Consider the TRS

$$
\mathcal{R}=\left\{\begin{array}{l}
\mathrm{f}(x, \mathrm{c}(\mathrm{a}), \mathrm{c}(\mathrm{b})) \rightarrow \mathrm{a} \\
\mathrm{f}(\mathrm{c}(\mathrm{b}), x, \mathrm{c}(\mathrm{a})) \rightarrow \mathrm{a} \\
\mathrm{f}(\mathrm{c}(\mathrm{a}), \mathrm{c}(\mathrm{b}), x) \rightarrow \mathrm{a}
\end{array}\right\}
$$

over the signature $\mathcal{F}$ consisting of all symbols appearing in the rewrite rules and the TRS $\mathcal{R}^{\prime}=\{\mathrm{g}(x) \rightarrow \mathrm{c}(x)\}$ over the signature $\mathcal{F}^{\prime}$ consisting of a constant $\mathrm{d}$ in addition to $\mathrm{g}$ and $\mathrm{c}$. Both TRSs have external normal forms, lack collapsing rules, and belong to $\mathcal{C} \mathcal{B N}_{\text {nv }}$. Their union does not belong to $\mathcal{C} \mathcal{B N}_{\text {nv }}$ as the term $\mathrm{f}(\mathrm{g}(\mathrm{a}), \mathrm{g}(\mathrm{a}), \mathrm{g}(\mathrm{a}))$ has no $\left(\mathcal{S}_{\text {nv }}, \mathcal{G}\right)$-needed redex. Note that $\mathcal{R}$ and $\mathcal{R}^{\prime}$ share the constructor $\mathrm{c}$ and hence $\mathrm{g}(x) \rightarrow \mathrm{c}(x)$ is constructor-lifting.

A simple modification of the above example shows the necessity of forbidding constructor-lifting rules in Theorem 5 even if we require that the constituent CSs lack external normal forms.

Example 9. Consider the TRSs

$$
\mathcal{R}=\left\{\begin{array}{ll}
\mathrm{f}(x, \mathrm{a}, \mathrm{b}) \rightarrow \mathrm{c}(\mathrm{g}(x)) & \mathrm{g}(x) \rightarrow \mathrm{g}(\mathrm{a}) \\
\mathrm{f}(\mathrm{b}, x, \mathrm{a}) \rightarrow \mathrm{c}(\mathrm{g}(x)) & \mathrm{h}(x) \rightarrow x \\
\mathrm{f}(\mathrm{a}, \mathrm{b}, x) \rightarrow \mathrm{c}(\mathrm{g}(x)) &
\end{array}\right\}
$$

and

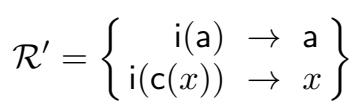

over the signatures $\mathcal{F}$ and $\mathcal{F}^{\prime}$ consisting of function symbols that appear in their respective rewrite rules. The two TRSs are obviously collapsing and share the constructors a and c. One easily verifies that both TRSs belong to $\mathcal{C} \mathcal{B N}_{\mathrm{nv}}$ and that $\operatorname{WN}\left(\mathcal{R}_{\mathrm{nv}}, \mathcal{G}, \mathcal{F}\right)=\operatorname{WN}\left(\mathcal{R}_{\mathrm{nv}}, \mathcal{F}\right)$ and $\operatorname{WN}\left(\mathcal{R}_{\mathrm{nv}}, \mathcal{G}, \mathcal{F}^{\prime}\right)=\mathcal{T}\left(\mathcal{F}^{\prime}\right)=$ $\mathrm{WN}\left(\mathcal{R}_{\mathrm{nv}}^{\prime}, \mathcal{F}^{\prime}\right)$. However, the union of the two TRSs does not belong to $\mathcal{C} \mathcal{B N}_{\mathrm{nv}}$ as the term $\mathrm{i}(\mathrm{f}(\Delta, \Delta, \Delta))$ with any collapsing redex $\Delta$ has no $\left(\mathcal{S}_{\text {nv }}, \mathcal{G}\right)$-needed redex.

\section{References}

1. F. Baader and T. Nipkow. Term Rewriting and All That. Cambridge University Press, 1998.

2. H. Comon. Sequentiality, monadic second-order logic and tree automata. Information and Computation, 157:25-51, 2000. 
3. N. Dershowitz. Orderings for term-rewriting systems. Theoretical Computer Science, 17:279-301, 1982.

4. N. Dershowitz and J.-P. Jouannaud. Rewrite systems. In Handbook of Theoretical Computer Science, volume B, pages 243-320. Elsevier, 1990.

5. I. Durand. Bounded, strongly sequential and forward-branching term rewriting systems. Journal of Symbolic Computation, 18:319-352, 1994.

6. I. Durand and A. Middeldorp. Decidable decidable call by need computations in term rewriting (extended abstract). In Proceedings of the 14 th International Conference on Automated Deduction, volume 1249 of LNAI, pages 4-18, 1997.

7. I. Durand and A. Middeldorp. On the complexity of deciding call-by-need. Technical Report 1194-98, LaBRI, Université de Bordeaux I, 1998.

8. B. Gramlich. Termination and Confluence Properties of Structured Rewrite Systems. PhD thesis, Universität Kaiserslautern, 1996.

9. G. Huet and J.-J. Lévy. Computations in orthogonal rewriting systems, I and II. In Computational Logic, Essays in Honor of Alan Robinson, pages 396-443. The MIT Press, 1991. Original version: Report 359, Inria, 1979.

10. F. Jacquemard. Decidable approximations of term rewriting systems. In Proceedings of the 7th International Conference on Rewriting Techniques and Applications, volume 1103 of $L N C S$, pages 362-376, 1996.

11. R. Kennaway, J.W. Klop, R. Sleep, and F.-J. de Vries. Comparing curried and uncurried rewriting. Journal of Symbolic Computation, 21(1):15-39, 1996.

12. J.W. Klop. Term rewriting systems. In Handbook of Logic in Computer Science, Vol. 2, pages 1-116. Oxford University Press, 1992.

13. J.W. Klop and A. Middeldorp. Sequentiality in orthogonal term rewriting systems. Journal of Symbolic Computation, 12:161-195, 1991.

14. A. Middeldorp. Modular Properties of Term Rewriting Systems. PhD thesis, Vrije Universiteit, Amsterdam, 1990.

15. T. Nagaya and Y. Toyama. Decidability for left-linear growing term rewriting systems. In Proceedings of the 10th International Conference on Rewriting Techniques and Applications, volume 1631 of LNCS, pages 256-270, 1999.

16. E. Ohlebusch. Modular Properties of Composable Term Rewriting Systems. PhD thesis, Universität Bielefeld, 1994.

17. M. Oyamaguchi. NV-sequentiality: A decidable condition for call-by-need computations in term rewriting systems. SIAM Journal on Computation, 22:114-135, 1993.

18. R. Strandh. Classes of equational programs that compile into efficient machine code. In Proceedings of the 3rd International Conference on Rewriting Techniques and Applications, volume 355 of LNCS, pages 449-461, 1989.

19. T. Takai, Y. Kaji, and H. Seki. Right-linear finite path overlapping term rewriting systems effectively preserve recognizability. In Proceedings of the 11th International Conference on Rewriting Techniques and Applications, volume 1833 of LNCS, pages 246-260, 2000.

20. Y. Toyama. Strong sequentiality of left-linear overlapping term rewriting systems. In Proceedings of the 7th IEEE Annual Symposium on Logic in Computer Science, pages 274-284, 1992.

21. Y. Toyama, S. Smetsers, M. van Eekelen, and R. Plasmeijer. The functional strategy and transitive term rewriting systems. In Term Graph Rewriting: Theory and Practice, pages 61-75. Wiley, 1993. 